\title{
Perfiles hemodinámicos de una serie de pacientes llevados a cateterismo cardíaco derecho. Experiencia de un centro de la ciudad de Medellín, Colombia
}

\author{
Hemodynamic profiles in a series of patients undergoing right heart catheterization. \\ Experience in one center of Medellin, Colombia
}

\author{
Orlando Castaño-Cifuentes ${ }^{1 *}$, Absalón A. Clavijo-Moldón ${ }^{2}$ y James S. Díaz-Betancur ${ }^{3}$ \\ ${ }^{1}$ Departamento de Cardiología, Universidad CES; ${ }^{2}$ Departamento de Medicina Interna; ${ }^{3}$ Departamento de Cardiología. Clínica Las Américas Auna. \\ Antioquia, Medellín, Colombia
}

\begin{abstract}
Resumen
Objetivo: El objetivo de este estudio fue caracterizar la clasificación hemodinámica de la hipertensión pulmonar en una serie de pacientes llevados a cateterismo cardíaco derecho. Métodos: Se realizó un estudio retrospectivo y se revisaron los registros de los cateterismos cardíacos derechos practicados en personas mayores de 18 años entre enero de 2017 y diciembre del 2018. Se excluyó a aquéllos con datos incompletos o no concluyentes y se definieron los perfiles hemodinámicos de acuerdo con el resultado de la presión en cuña y la resistencia vascular pulmonar. Resultados: Se incluyó a 92 sujetos de los cuales se descartó hipertensión pulmonar en 4 . El $26.1 \%$ se clasificó como precapilar, $31.8 \%$ como poscapilar y $42 \%$ como combinada. No hubo diferencias entre los tres grupos en edad y sexo; las presiones pulmonares fueron más elevadas en el grupo de hipertensión pulmonar precapilar. Se encontró presión en cuña elevada en el $73.8 \%$ de los pacientes y el cateterismo derecho reclasificó al $35 \%$ de los pacientes en un grupo hemodinámico diferente al esperado. En la mayor parte de estos casos se sospechaba HP precapilar por el diagnóstico anterior al procedimiento, pero los perfiles hemodinámicos encontrados correspondieron a hipertensión poscapilar o combinada. Conclusión: El cateterismo cardíaco derecho es necesario para la clasificación hemodinámica de la hipertensión pulmonar, que es importante no sólo para el diagnóstico sino para evaluar la causa de la enfermedad.
\end{abstract}

Palabras clave: Cateterismo cardíaco. Clasificación. Hemodinámica. Hipertensión pulmonar. Presión en cuña pulmonar.

\begin{abstract}
Objective: To characterize the hemodynamic classification of pulmonary hypertension in a series of patients undergoing to right cardiac catheterization. Methods: This is a retrospective study, we analyze the records of all right heart catheterizations performed between January 2017 and December 2018, all patient older than 18 years were included and those with incomplete or inconclusive data were excluded. We identify three hemodynamic profiles according to the result of right heart catheterization. Results: 92 subjects were included and pulmonary hypertension was ruled out in 4 of them; $26.1 \%$ were classified as precapillary, $31.8 \%$ postcapillary and $42 \%$ as combined pulmonary hypertesion. There were no differences between the 3 groups in age or sex, pulmonary pressures were higher in the precapillary pulmonary hypertension group, right
\end{abstract}

Correspondencia:

*Orlando Castaño-Cifuentes

E-mail: orca182@ hotmail.com

1405-9940 / @ 2021 Instituto Nacional de Cardiología Ignacio Chávez. Publicado por Permanyer. Este es un artículo open access bajo la licencia
Fecha de recepción: 23-11-2020

Fecha de aceptación: 17-04-2021
Disponible en internet: 03-01-2022

www.archivoscardiologia.com
Arch Cardiol Mex. 2022;92(1):53-59

CC BY-NC-ND (http://creativecommons.org/licenses/by-nc-nd/4.0/) 
catheterization reclassified $35 \%$ of the patients in a hemodynamic group in a different from that expected. Precapillary PH was suspected in most of these cases, but the hemodynamic profiles found in cardiac catheterization corresponded to post-capillary or combined pulmonay hypertension. Conclusion: Right heart catheterization is necessary for the hemodynamic classification of pulmonary hypertension, which is important not only for diagnosis but also for evaluating the etiology of the disease.

Keywords: Cardiac catheterization. Classification. Hemodynamics. Pulmonary hypertension. Pulmonary wedge pressure.

\section{Introducción}

La hipertensión pulmonar (HP) se define como la presión de la arteria pulmonar media (PAPm) $>20 \mathrm{mmHg}$ cuantificada por cateterismo cardíaco derecho durante el reposo ${ }^{1}$. Este punto de corte es menor al recomendado en las definiciones previas $^{2} y$, aunque carece de evidencia científica, tiene una razón fisiológica. El cambio del umbral diagnóstico para HP se realizó para reconocer de forma temprana la enfermedad y permitir intervenciones tempranas que mejoren el pronóstico y la calidad de vida de los pacientes. Por otro lado, se debe señalar que los términos HP e hipertensión arterial pulmonar (HAP) no son indistintos; el primero hace referencia a un valor hemodinámico mientras que el segundo representa un síndrome clínico ${ }^{3}$.

Los datos epidemiológicos muestran que la HP tiene una prevalencia de 97 casos por 1 millón de habitantes y la edad promedio de diagnóstico es de 50 a 65 años ${ }^{2}$. La HP del grupo 2 es la más frecuente y se halla entre el $60 \%$ y el $70 \%$ de los casos $^{4,5}$. En cuanto al pronóstico de la enfermedad, se ha establecido que la mortalidad de los pacientes depende de la clasificación del riesgo ${ }^{6}$ y los registros clínicos han confirmado el mal pronóstico de la HAP7.

En Latinoamérica se cuenta con registros de diferentes países, pero la falta de definiciones estandarizadas dificulta establecer datos epidemiológicos precisos y es todavía una enfermedad subdiagnosticada ${ }^{8-10}$. Aunque se han desarrollado varios registros clínicos sobre HAP, hay poca información sobre los otros grupos de HP. Las guías de práctica clínica recomiendan un diagnóstico temprano y una adecuada clasificación de la $\mathrm{HP}^{11}$ para iniciar tratamiento inmediato e individualizado. El cateterismo cardíaco derecho es la prueba regular para el diagnóstico y permite clasificar la HP como precapilar, poscapilar o combinada; estos tres se conocen como perfiles hemodinámicos. La HP precapilar corresponde a los pacientes de los grupos 1, 3, 4 y algunos del grupo 5 de la clasificación de HP, mientras que la HP poscapilar incluye al grupo 2 que comprende a los pacientes con insuficiencia cardíaca, lesiones valvulares cardíacas y algunas lesiones obstructivas poscapilares ${ }^{5}$. El tercer perfil hemodinámico denominado HP combinada puede incluir a la HP de diferentes grupos y algunas formas de falla cardíaca avanzada requieren análisis más cuidadoso y parecen tener un comportamiento particular ${ }^{12}$. Aunque en la práctica clínica la mayoría de los pacientes con HP no requiere un cateterismo cardíaco, una vez efectuada esta prueba las variables hemodinámicas encontradas pueden confirmar la causa de la HP o sugerir causas diferentes a las esperadas antes del procedimiento, según sea el perfil hemodinámico encontrado. Los autores realizaron este estudio para caracterizar los perfiles hemodinámicos de HP en una serie de pacientes llevados a cateterismo cardíaco derecho en un centro de referencia.

\section{Métodos}

Tipo de estudio: se realizó un estudio retrospectivo en la Clínica Las Américas Auna, un centro de referencia para pacientes con enfermedades cardiovasculares ubicado en Medellín, Colombia.

Población y obtención de la información: se revisaron los registros de todos los cateterismos cardíacos derechos practicados en pacientes mayores de 18 años entre enero de 2017 y diciembre del 2018. Se excluyó a aquéllos con datos incompletos o no concluyentes. En caso de que un mismo individuo fuera objeto de varios cateterismos durante el período del estudio, se tuvieron en cuenta todos los registros para la correlación entre la presión sistólica de la arteria pulmonar (PSAP) determinada por ecocardiografía y cateterismo, pero sólo se consideró el primer estudio durante el lapso de tiempo indicado para caracterizar los perfiles hemodinámicos. El gasto cardíaco se calculó por termodilución y se utilizó el método de Fick en pacientes con insuficiencia valvular tricuspídea moderada a grave. Una sola persona recopiló la información en un archivo digital.

Análisis estadísticos: se definió la hipertensión pulmonar (HP) como una presión media de la arteria pulmonar (PAPm) >20 mmHg mensurada con cateterismo 
cardíaco derecho ${ }^{1}$. Entre los pacientes con HP se definieron tres perfiles hemodinámicos de acuerdo con el resultado de la presión en cuña o presión capilar pulmonar (PCP) y la resistencia vascular pulmonar (RVP). En consecuencia, se definió la HP precapilar como una $\mathrm{PCP} \leq 15 \mathrm{mmHg}$ y RVP $\geq 3$ unidades Wood, la HP poscapilar como $\mathrm{PCP}>15 \mathrm{mmHg}$ y RVP $<3$ unidades Wood y la HP combinada como PCP $>15 \mathrm{mmHg}$ y RVP $\geq 3$ unidades Wood. Las variables cualitativas se describieron en valores absolutos y relativos y las variables cuantitativas con medianas y rango intercuartílico (RIC) porque no cumplieron el supuesto de distribución normal. Para comparaciones entre variables cualitativas se utilizó la ji cuadrada o la prueba exacta de Fisher cuando fue necesario y para comparaciones entre variables cuantitativas se utilizó la prueba de Kruskal-WaIlis. Los análisis estadísticos se efectuaron con el software Stata versión 14.2 y se consideró un valor de $p<0.05$ como estadísticamente significativo.

\section{Resultados}

Durante el período de estudio se realizaron $100 \mathrm{ca}-$ teterismos cardíacos derechos. Se excluyeron tres registros porque las variables hemodinámicas no fueron concluyentes; uno de estos registros correspondió a un paciente con insuficiencia tricuspídea grave y otro a un paciente con una comunicación interauricular $(\mathrm{CIA})$. Quedaron 97 cateterismos cardíacos derechos en 92 personas que se incluyeron en el análisis. En la Figura 1 se presentan las indicaciones para solicitud del procedimiento. La principal indicación para el cateterismo derecho fue la sospecha de hipertensión pulmonar por resultado de ecocardiografía (52\%), el estudio de cardiopatías congénitas (16\%) y la valoración de la posibilidad de trasplante cardíaco en pacientes con insuficiencia cardíaca (13\%). El 19\% restante de los cateterismos se solicitó para tamizaje de hipertensión pulmonar en pacientes con enfermedad reumatológica, seguimiento de hipertensión pulmonar en individuos con la enfermedad o para estudio de valvulopatías cardíacas en la minoría de los casos (Fig. 1).

Prevalencia de HP: se descartó HP en 4 de las 92 personas, de tal modo que quedaron al final 88 pacientes con diagnóstico positivo (95.6\%). Estos individuos se clasificaron como HP precapilar $(n=23,26.1 \%)$, HP poscapilar $(n=28,31.8 \%)$ o HP combinada $(n=37$, $42 \%$ ) de acuerdo con el resultado de la PCP y la RVP. La mayoría de los enfermos con HP tuvo PCP elevada $(n=65,73.8 \%)$

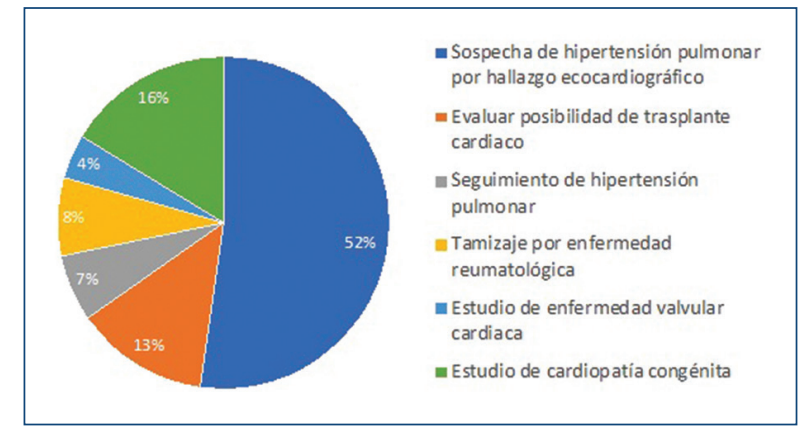

Figura 1. Indicaciones para el cateterismo cardíaco derecho $(n=92)$.

Características clínicas y hemodinámicas: En la Tabla 1 se describen las características de los pacientes en relación con el perfil hemodinámico. No se encontraron diferencias significativas entre las medianas de edad ni sexo entre los tres grupos. Aunque las medianas de la fracción de expulsión del ventrículo izquierdo ( $\mathrm{FEVI}$ ) fueron similares, ningún paciente con HP precapilar tuvo $\mathrm{FEVI}<40 \%$ y un alto porcentaje de pacientes en los grupos de HP poscapilar e HP combinada registró una $\mathrm{FEVI}>50 \%$. La disfunción ventricular derecha definida como dilatación del ventrículo derecho descrita en la ecocardiografía estuvo presente en $64.3 \%$ de los pacientes con HP poscapilar, $37.8 \%$ de los enfermos con HP combinada y $22.7 \%$ de los pacientes con HP precapilar. Por su parte, las medianas de PSAP, presión diastólica de la arteria pulmonar y PAPm fueron significativamente más elevadas en los individuos con HP precapilar respecto del grupo con HP poscapilar (Tabla 1).

Reclasificación hemodinámica: la indicación para cateterismo derecho fue cardiopatía congénita en 14 pacientes y otras anomalías diferentes de la cardiopatía congénita en 74 pacientes. En total, se encontró un perfil hemodinámico distinto al esperado en 31 de los $88(35.2 \%)$ pacientes con HP.

En 12 de los 14 enfermos con cardiopatía congénita (85.7\%), la clasificación hemodinámica fue diferente a la HP precapilar que es el perfil esperado: 7 correspondieron a HP combinada y 5 a HP poscapilar; estos últimos tenían diagnóstico de CIA y comunicación interventricular (CIV). En 19 de los 74 pacientes con indicación diferente a la cardiopatía congénita (25.7\%), el cateterismo demostró una clasificación hemodinámica distinta de la esperada. En la mayor parte de estos casos se sospechaba HP precapilar por enfermedades como tromboembolia pulmonar crónica, enfermedad 
Tabla 1. Características de los pacientes de acuerdo con el perfil hemodinámico

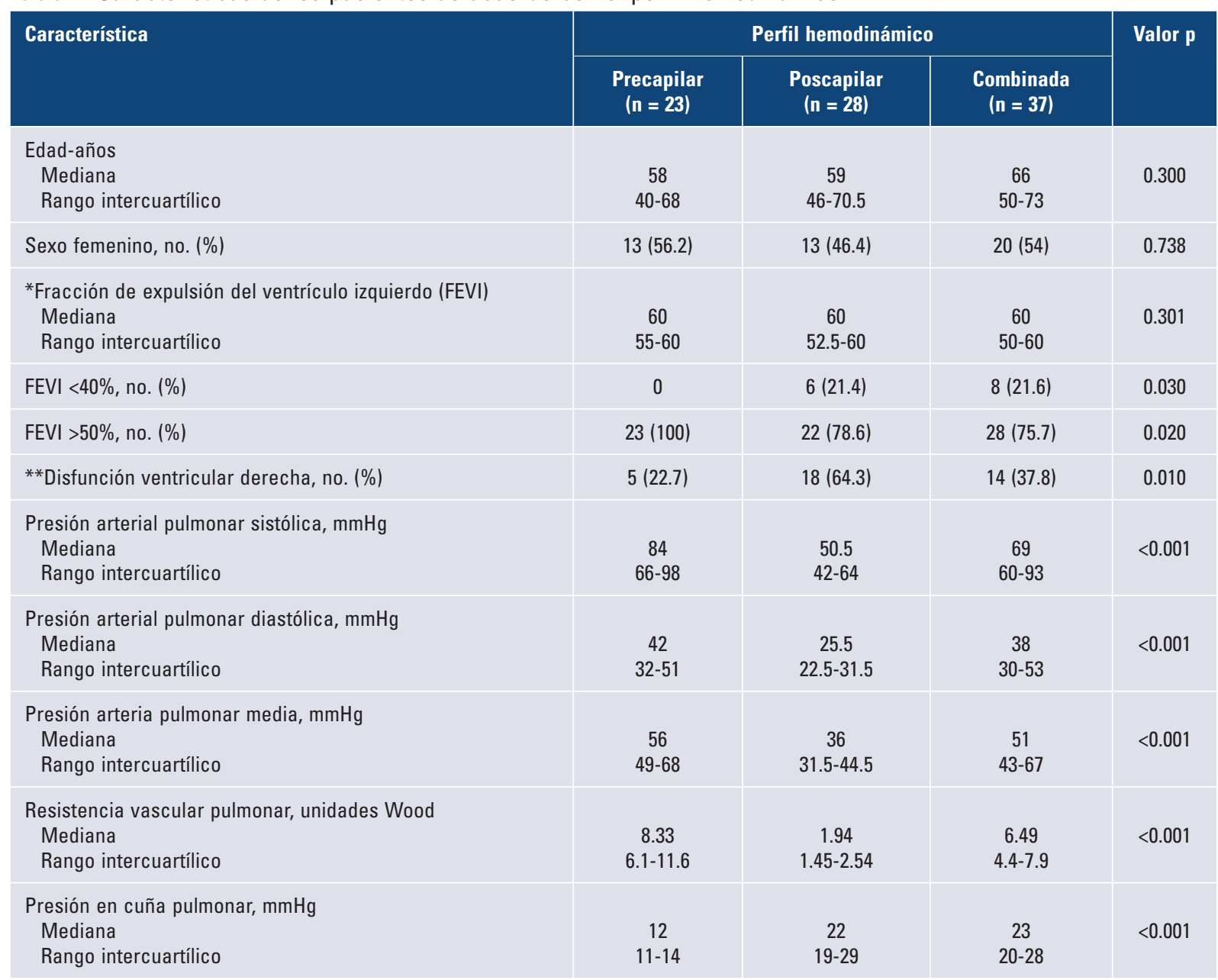

FEVI = fracción de expulsión del ventrículo izquierdo.

*Fracción de expulsión del ventrículo izquierdo determinada por ecocardiografía.

**Definida como dilatación del ventrículo derecho en ecocardiografía.

pulmonar obstructiva crónica, enfermedad pulmonar intersticial, fibrosis pulmonar, lupus y artritis reumatoide. Sin embargo, el perfil hemodinámico identificado en el cateterismo los clasificó como HP poscapilar o HP combinada. Sólo en 1 de los 19 pacientes la HP se clasificó como precapilar cuando antes del procedimiento se sospechaba que era poscapilar por insuficiencia cardíaca.

Características de los pacientes con HP combinada: después de excluir a los sujetos con cardiopatía congénita, 30 pacientes se clasificaron con HP combinada. Se identificó $\mathrm{FEVI}$ preservada (FEVI $>50 \%$ ) en 21 de ellos (70\%) y varios tenían comorbilidades como tromboembolia pulmonar crónica, enfermedad pulmonar intersticial y síndrome de apnea obstructiva del sueño.
Correlación ecocardiográfica con hallazgos del cateterismo derecho: en la Figura 2 se presenta la correlación entre la PSAP calculada por ecocardiografía y la medida durante el cateterismo cardíaco, con coeficiente de correlación de Spearman de 0.63 y $\mathrm{p}<0.001$.

\section{Discusión}

En este estudio se presentan los perfiles hemodinámicos de una serie de individuos llevados a cateterismo cardíaco derecho, la mayoría de ellos con sospecha de hipertensión pulmonar por tamizaje ecocardiográfico. Se encontró una alta proporción de pacientes en los que la clasificación hemodinámica encontrada durante el procedimiento fue diferente a la esperada según el diagnóstico anterior a él. Este hallazgo resalta 


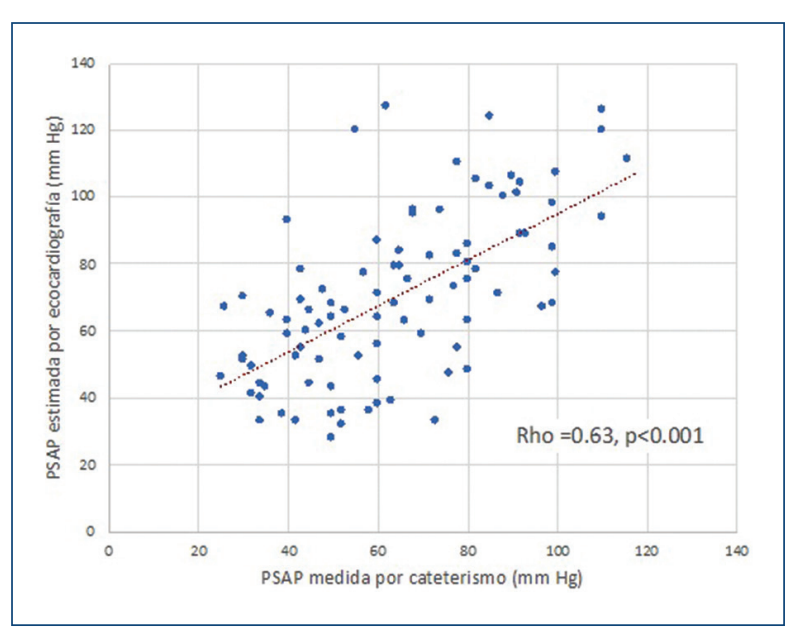

Figura 2. Correlación entre la PSAP calculada por ecocardiografía y la PSAP medida con cateterismo $(n=92)$.

la importancia de tener un bajo umbral para solicitar cateterismo cardíaco, en particular cuando hay sospecha de una causa mixta de la HP.

Aunque los datos epidemiológicos muestran que la HP tiene una prevalencia de apenas 97 casos por cada millón de habitantes ${ }^{2}$, más del $95 \%$ de los individuos llevados a cateterismo cardíaco derecho en este estudio tenía HP según la definición actual. Esto demuestra la baja utilización de este recurso diagnóstico en el medio y el diagnóstico tardío de la enfermedad, aunque incluso las publicaciones regionales reconocen el mal pronóstico de la $\mathrm{HP}^{7}$. Otra publicación mostró una utilización del cateterismo derecho en apenas $40 \%$ de una serie de pacientes con sospecha de hipertensión pulmonar tromboembólica ${ }^{10}$.

Los autores también observaron una gran correlación entre la PSAP calculada por ecocardiografía y la PSAP medida en el cateterismo derecho. Esto ya se conoce bien ${ }^{13}$, pero es importante recordar que la variabilidad en las mediciones ecocardiográficas hacen que este método no sea confiable ${ }^{14} \mathrm{y}$ sólo se recomienda para estratificar la probabilidad de $\mathrm{HP}^{11}$. Para el estudio de la HP es necesario practicar el cateterismo cardíaco como lo recomiendan las guías de práctica clínica²; este procedimiento, además de confirmar la enfermedad, permite obtener las variables hemodinámicas con las cuales es posible determinar la causa de la HP en los casos con componente poscapilar adjunto, es decir, con PCP, al margen del diagnóstico, y tiene implicaciones terapéuticas y para el pronóstico. En consecuencia, por ejemplo, un paciente con hipertensión pulmonar precapilar requiere tratamiento vasodilator específico, mientras que un paciente con hipertensión pulmonar poscapilar aislada obliga al clínico a evaluar la posibilidad de insuficiencia cardíaca, sobre todo con FEVI preservada, una enfermedad que requiere un enfoque por completo diferente. Además, en la actualidad se considera que los pacientes clasificados como HP combinada, gracias a los resultados del cateterismo derecho, podrían tener un comportamiento diferente y en algunos casos beneficiarse de los tratamientos vasodilatadores ${ }^{15}$.

Por otro lado, en esta serie se identificó una dilatación ventricular derecha en casi 1 de cada 5 pacientes con HP. Un registro latinoamericano de HAP notificó disfunción grave del ventrículo derecho en 1 de cada 3 pacientes ${ }^{16}$. La prevalencia de disfunción ventricular derecha en estudios clínicos varía entre $8 \%$ y $10 \%{ }^{17}$ y revela el retraso diagnóstico en este medio. De manera llamativa, la dilatación del ventrículo derecho en este estudio fue más frecuente en los pacientes con HP poscapilar, si bien los enfermos con HP precapilar e HP combinada tenían medianas de PAPm y RVP significativamente mayores. Esto pone de manifiesto que los mecanismos de daño ventricular derecho en la HP son multifactoriales y que existen factores independientes de la presión ${ }^{18}$. Además, la disfunción ventricular izquierda en la HP poscapilar podría influir en la función ventricular derecha.

Los autores observaron una alta proporción de pacientes con HP y PCP elevada (73.8\%), lo que refleja la presencia de disfunción ventricular izquierda como causa o factor contribuyente a la HP. La más reciente guía de práctica clínica para el diagnóstico de la insuficiencia cardíaca con FEVI preservada subraya la importancia de la medición hemodinámica para confirmar el diagnóstico de la enfermedad cuando no se ha establecido el diagnóstico con el péptido natriurético ni con la ecocardiografía ${ }^{19}$. La gran frecuencia de PCP elevada en este estudio demuestra que no es posible subestimar la disfunción ventricular izquierda como causa de la HP en esta población ni considerar sobre todo esta entidad antes que otros orígenes de la HP. La HP combinada se ha descrito de manera amplia en estudios observacionales e incluso se ha planteado la posibilidad de un continuo entre HAP y HP secundaria a insuficiencia cardíaca ${ }^{15}$; en este estudio se halló una prevalencia del $42 \%$ de esta entidad, que fue más frecuente en los individuos con FEVI preservada junto con comorbilidades.

El hallazgo más significativo de esta investigación fue la alta frecuencia de reclasificación hemodinámica 
de la HP y el perfil hemodinámico identificado en el cateterismo fue diferente al esperado según el diagnóstico anterior al procedimiento en $35.2 \%$ de los pacientes. La reclasificación hemodinámica en los pacientes con cardiopatías congénitas del tipo CIA y CIV se explica con facilidad porque este tipo de defectos induce sobrecarga de volumen en las cavidades cardíacas izquierdas y por tanto compromiso pulmonar poscapi$\operatorname{lar}^{20}$. Empero, la reclasificación también se observó en pacientes con otras indicaciones para cateterismo derecho. En un registro previo, hasta el $37 \%$ de los pacientes llevados a cateterismo derecho tenía un diagnóstico diferente al inicial y el $52 \%$ de los pacientes llevados a cateterismo derecho e izquierdo tuvo diagnóstico distinto al inicial ${ }^{21}$.

Si bien el cateterismo derecho es un procedimiento invasivo, la mayor parte de las complicaciones comunicadas se deriva de series de casos y su verdadera incidencia se desconoce; sin embargo, los estudios de la década de 1980 registraron una mortalidad hasta del $5.3 \%$, pero en fecha más reciente los informes sólo muestran una incidencia del $0.05 \%$ y se han descrito con más frecuencia cuando no se usa fluoroscopia para guiar el paso del catéter ${ }^{22}$. Aunque las complicaciones son raras, incluyen situaciones tan graves como ruptura de cámaras cardíacas derechas, ruptura de la arteria pulmonar, daño valvular, embolización por ruptura del catéter, complicaciones vasculares en la inserción del catéter e incluso endocarditis infecciosa ${ }^{23}$. En este estudio no se presentaron complicaciones, lo que sugiere que es un procedimiento seguro en manos de personal experto.

Los resultados remarcan la importancia de efectuar el cateterismo cardíaco derecho, no sólo para comprobar el diagnóstico de HP, sino para establecer la causa, estratificar el riesgo de manera precisa y ofrecer tratamiento individualizado. Aunque es claro que el cateterismo derecho no es necesario para la mayor parte de los grupos de HP, este trabajo permite recomendar un bajo umbral para solicitar el procedimiento, en especial cuando hay dudas sobre el origen de la HP después de interpretar los resultados de las pruebas no invasivas como la ecocardiografía y los péptidos natriuréticos, o cuando se sospecha una causa mixta de la HP según el contexto clínico del paciente. La adecuada clasificación hemodinámica podría modificar el pronóstico de la enfermedad ${ }^{24}$, más cuando hasta en el $57 \%$ de los casos los tratamientos ofrecidos a pacientes con HP no concuerdan con los recomendados por las guías de práctica clínica ${ }^{21,25,26}$.
Como limitaciones debe mencionarse que se incluyó a un centro único y los sujetos fueron referidos, por lo que los resultados no representan a la población general ni pueden extrapolarse a ella.

\section{Conclusiones}

El cateterismo cardíaco derecho es necesario no sólo para el diagnóstico de la HP sino para su clasificación hemodinámica. La valoración hemodinámica es importante para determinar de manera precisa la causa de la enfermedad y puede llevar a reconsiderar tal causa en una elevada proporción de los casos.

\section{Financiamiento}

Ninguno.

\section{Conflicto de intereses}

Ninguno.

\section{Responsabilidades éticas}

Protección de personas y animales. Los autores declaran que para esta investigación no se han realizado experimentos en seres humanos ni en animales.

Confidencialidad de los datos. Los autores declaran que han seguido los protocolos de su centro de trabajo sobre la publicación de datos de pacientes.

Derecho a la privacidad y consentimiento informado. Los autores declaran que en este artículo no aparecen datos de pacientes.

\section{Bibliografía}

1. Simonneau G, Montani D, Celermajer DS, Denton CP, Gatzoulis MA, Krowka M, et al. Haemodynamic definitions and updated clinical classification of pulmonary hypertension. The European Respiratory Journal. 2019;53(1).

2. Galiè HM, Vachiery JL, Gibbs S, Lang I, Torbicki A, Simonneau G, et al. ESC Scientific Document Group. 2015 ESC/ERS Guidelines for the diagnosis and treatment of pulmonary hypertension: The Joint Task Force for the Diagnosis and Treatment of Pulmonary Hypertension of the European Society of Cardiology (ESC) and the European Respiratory Society (ERS): Endorsed by: Association for European Paediatric and Congenital Cardiology (AEPC), International Society for Heart and Lung Transplantation (ISHLT). Eur Heart J. 2016 Jan 1;37(1):67-119.

3. Simonneau GM, Adatia I, Celermajer D, Denton C, Ghofrani A, Gómez-Sánchez MA, et al. Updated clinical classification of pulmonary hypertension. J Am Coll Cardiol. 2013 Dec 24;62((25 Suppl):):D34-41.

4. Badesch $\mathrm{CH}$, Gómez-Sánchez MA, Hoeper M, Loyd J, Manes A McGoon M, et al. Diagnosis and assessment of pulmonary arterial hypertension. J Am Coll Cardiol. 2009;54(Suppl):S55-S6.

5. Vachiery JL, Tedford RJ, Rosenkranz S, Palazzini M, Lang I, Guazzi M, et al. Pulmonary hypertension due to left heart disease. The European Respiratory Journal. 2019;53(1).

6. Hoeper MM, Kramer T, Pan Z, Eichstaedt CA, Spiesshoefer J, Benjamin N, et al. Mortality in pulmonary arterial hypertension: prediction by the 2015 European pulmonary hypertension guidelines risk stratification model. The European Respiratory Journal. 2017;50(2). 
7. Valverde $A B$, Viana KP, Gomes B, Soares $C$, Souza R. Pulmonary arterial hypertension in Latin America: epidemiological data from local studies. BMC Pulmonary Medicine. 2018;18:106.

8. Miranda PA, Guzmán-Sáenz RC, Baños IA. Epidemiologia de la hipertension pulmonar en Colombia. Salud Uninorte Barranquilla (Col). 2018;34(3):607-24.

9. Miranda-Machado P, Banos-Álvarez I, Álvarez-Barrios A. Prevalence of pulmonary arterial hypertension in the Colombian Caribbean. Pulmonary Circulation. 2019;9(2):2045894019847643.

10. Conde VC, Duenas R, Torres A. Diagnosis and treatment of pulmonary arterial hypertension and chronic thromboembolic pulmonary hypertension in five reference centers. In Bogota-Colombia, at 2.640 meters above sea level. Am J Respir Crit Care Med. 2015;191:A4847.

11. Frost A, Badesch D, Gibbs JSR, Gopalan D, Khanna D, Manes A, et al Diagnosis of pulmonary hypertension. The European Respiratory Journal. 2019;53(1).

12. Ibe T, Wada H, Sakakura K, Ugata Y, Yamamoto K, Seguchi M, et al Combined pre- and post-capillary pulmonary hypertension defined by new criteria is worse prognosis group in patients with heart failure, European Heart Journal. 2020;41(Suppl 2):946-2285

13. Harrison W, Farber AJF, Miller DP, McGoon MD. REVEAL Registry: correlation of right heart catheterization and echocardiography in patients with pulmonary arterial hypertension. Congest Heart Fail. 2011;17(2):56-64.

14. Augustine DX, Coates-Bradshaw LD, Willis J, Harkness A, Ring L, and the British Society of Echocardiography Education Committee. Echocardiographic assessment of pulmonary hypertension: a guideline protocol from the British Society of Echocardiography. Echo Res Pract. 2018;5(3):G11-G24.

15. Opitz CF, Gibbs JSR, Kaemmerer H, Pepke-Zaba J, Coghlan JG, Scelsi L, et al. Pre-capillary, combined, and post-capillary pulmonary hypertension: a pathophysiological continuum. J Am Coll Cardiol. 2016:68(4):368-78.

16. Coronel ML, Núñez C, Cursack G, Fleitas M, Botta C. Severe right ventricular dysfunction in pulmonary arterial hypertension: prevalence, clinical markers and treatment in Argentinean HINPULSAR registry. Eur $\mathrm{J}$ Heart Fail. 2014;16:293-4.
17. Sitbon O, Channick R, Chin KM, Frey A, Gaine S, Galiè N, et al., for the GRIPHON Investigators. Selexipag for the treatment of pulmonary arterial hypertension. N Engl J Med. 2015;373:2522-33.

18. Ren X. Right heart in pulmonary hypertension: from adaptation to failure. Pulmonary Circulation. 2019;9(3):1-20.

19. Pieske B, de Boer RA, Fraser AG, Anker SD, Donal E, Edelmann F, et al. How to diagnose heart failure with preserved ejection fraction: the HFAPEFF diagnostic algorithm: a consensus recommendation from the Heart Failure Association (HFA) of the European Society of Cardiology (ESC). European Heart Journal. 2019;40(40):3297-317

20. Rosenkranz S, Preston IR. Right heart catheterisation: best practice and pitfalls in pulmonary hypertension. european respiratory review: an official Journal of the European Respiratory Society. 2015;24(138):642-52.

21. Deaño RC, Rubenfire M, Frost A, Visovatti S, McLaughlin VV, Gomberg-Maitland M. Referral of patients with pulmonary hypertension diagnoses to tertiary pulmonary hypertension centers: the multicenter RePHerral study. JAMA Intern Med. 2013 173(10):887-93.

22. D'Alto M, Dimopoulos K, Coghlan JG, Kovacs G, Rosenkranz S, Naeije R. Right heart catheterization for the diagnosis of pulmonary hypertension: controversies and practical issues, Heart Fail Clin. 2018 Jul;14(3):467-477.

23. Chen Y, Shlofmitz E, Khalid N, Bernardo N, Ben-Dor I, Weintraub WS, et al. Right heart catheterization-related complications. A review of the literature and best practices. Cardiology in Review. 2020;28(1):36-41.

24. Conde-Camacho R, Cabrales-Arévalo J. El cateterismo derecho como herramienta en el diagnóstico de la hipertensión pulmonar. Revista Colombiana de Cardiología. 2017;24:28-33.

25. Sitbon O, Gomberg-Maitland M, Granton J, Lewis MI, Mathai SC, Rainisio M, et al. Clinical trial design and new therapies for pulmonary arterial hypertension. The European Respiratory Journal. 2019;53(1).

26. Garrido-Lestache MEB, Gómez-Sánchez MÁ. Estrategia terapéutica en hipertensión arterial pulmonar. Revista Colombiana de Cardiología. 2017;24:41-7. 